Notre Dame Journal of Formal Logic

Volume XVIII, Number 3, July 1977

NDJFAM

\title{
A REASSESSMENT OF GEORGE BOOLE'S THEORY OF LOGIC
}

\author{
JAMES W. VAN EVRA
}

George Boole's theory of logic has not fared well at the hands of the commentators who have written about it, whether they be his successors in logic itself, or historians of the subject. While there is general agreement that his work occupies an important place in the history of logic, the exact nature of that importance remains elusive. On the one hand, he has been called the originator of mathematical logic, ${ }^{1}$ but on the other, that claim has been pointedly disputed. ${ }^{2}$ On the one hand, his logic does differ significantly from traditional syllogistic logic, and for this he has been applauded. ${ }^{3}$ But on the other, Frege's introduction of quantification theory forms such a complete barrier between paleo- and neologic that any lasting influence from Boole's work, if it is there at all, seems permanently obscured.

Fueling these general concerns about the significance of Boole's work are the many claims that errors abound in it. These center mainly on his supposedly uncritical use of mathematics in logical contexts, which, so the critics suggest, resulted in the appearance of logically uninterpretable expressions in his system of logic. Not surprisingly, many of these same critics suggest that Boole's successors in algebraic logic put things right by providing logical interpretations for these expressions, thus extending the symmetry between logic and mathematics.

Here are a few examples of the sort of criticism I have in mind:

... Boole's quasi-mathematical system [can] hardly be regarded as a final and unexceptionable solution of the problem [of supplying a viable alternative to Aristotelian logic]. Not only did it require the manipulation of mathematical symbols in a very intricate and perplexing manner, but the results when obtained were devoid of demonstrative force, because they turned upon the employment of unintelligible symbols, acquiring meaning only by analogy. ${ }^{4}$

[The] mathematical character [of his work] is responsible at once for the strength and the weakness of Boole's calculus, as on the one hand, it could hardly have assumed so general a form had not Boole been able throughout to 
utilise the rules and operations which hold good in a $0-1$-algebra, while on the other hand, the mathematical method renders the operations effected and the expressions arising in dealing with the problems more or less obscure, since they cannot be subjected to logical interpretation. ${ }^{5}$

Boole's mathematicism goes so far ... that he introduces symbols and procedures which admit of no logical interpretation, or only a complicated and scarcely interesting one. Thus we meet with subtraction and division and numbers greater than $1 .^{6}$

Although Boole's system lends itself in some ways to easy manipulation, we must admit that it contains . . . some defects of rigour such as the use of the letter $v$ for the expression of existential propositions, the admission of numerical coefficients other than 1 and 0 , and the use of the operation of division to which no constant meaning has been assigned in logic. In the course of the half century after the publication of his Laws of Thought these shortcomings were all removed by his followers. ${ }^{7}$

L'algèbre de Boole souffrait d'un certain nombre de défauts. Avant tout elle comprenait des expressions énigmatiques, par exemple les coefficients $0 / 0$ ou $1 / 0 \ldots$ ou encore le coefficient $2 / 3$ qui s'y recontre de temps à autre, etc. $^{8}$

Owing to his free use of algebraic operations such as division and subtraction, the success of Boole's general method is offset by the appearance, in his calculations, of numerical and fractional coefficients and of other expressions whose logical significance is, to say the least, uncertain. It was the task of his successors to get rid of these anomalies and so to give the system a greater appearance of being what it claimed, a mathematical logic and not the mere logical mathematics that its opponents suspected it to be. ${ }^{9}$

The view of Boole's theory of logic which I present below centers on the following points:

First, specific criticisms such as those above, i.e., those to the effect that Boole includes logically uninterpretable expressions in his system of logic, are largely undeserved. They turn on a misunderstanding of what Boole called his "general method in logic," which provides for the inclusion of purely mathematical interludes in logical transformations, as a way of putting the formal power of mathematics to work in logical contexts. The questionable expressions serve as components in such interludes only, and were not considered by Boole to be logical expressions at all.

Concentrating on the general method, there is also a way of squaring Boole's explicit rejection of division as a logical operation, with his apparent (implicit) acceptance of the operation as a basis for interpreting coefficients such as $0 / 0$ and $1 / 0$. Though the evidence is largely circumstantial, it is possible that Boole did not count such expressions as being logical either, but again as being parts of the general method only.

Second, suggestions to the effect that Boole's successors in algebraic logic corrected his "mistakes" are misleading. Later algebraic logics were indeed more systematic, complete, and generally understandable than Laws of Thought (hereinafter ' $L T$ '), but in an important sense, they remain inferior to it. Specifically, Boole's successors tended uncritically to assume that logic and algebra fit perfectly together in the sense that they 
share all of their operations. But that assumption creates genuine problems for logic, the main one being, ironically, the unintelligibility of division used as a logical operator. While Boole's logic is far from perfect, he displays a greater awareness of logic as an independent discipline, as well as more sophistication in the use of mathematics as an aid to logic while avoiding the conflation of the two areas, than did his successors in algebraic logic.

Third, much of the relatively recent criticism of Boole's logic treats him as if he were a contemporary logician. But taking him to task for not, e.g., providing the same sophistication in proofs as one finds in contemporary logic not only overlooks the plain fact that Boole stands on the other side of a tremendous revolution in logic, it obscures much of his true importance in the history of the subject. That importance, it seems to me, is best exemplified by his general method. Regardless of its imperfection by today's standards, Boole's use of an independent formal device as an aid to logical processes marks him as an important figure in the transitional epoch between the traditional, purely syllogistic logics, and the contemporary, post-Fregean logics.

The following discussion deals exclusively with Boole's theory as it occurs in LT. After its publication, Boole wrote an essay (in [2], pp. 211ff.) containing views which radically diverge from those contained in LT. However, the status of that essay remains unclear, and there is evidence that Boole never adopted the views it contains. ${ }^{10}$

1 Even a cursory glance at LT reveals that the formal developments it contains occur in two separate sections of the work. On the one hand, Chapter II contains the basic logic, i.e., the fundamental logical system. On the other hand, Chapters V-VIII deal with the process of development, and its subsidiaries, such as elimination and reduction. The remainder of the logical portion of the work (which comprises the first fifteen chapters) is devoted to a variety of essentially auxiliary topics, such as variant interpretations of the symbolism, and specific examples of inferences using the logic already developed.

Boole apparently considered the separation between these two parts of the work to have more than merely spatial significance. As will be seen below, he considered the later chapters to be dependent on the basis laid in Chapter II, but at the same time to be more than just a simple extension of it. The two parts were, for Boole, of two fundamentally different sorts.

Chapter II is entitled "Of Signs in General, and of the Signs Appropriate to the Science of Logic in Particular; also of the Laws to which that Class of Signs are Subject." As the title suggests, the chapter consists of a virtually self-contained presentation of Boole's basic logic. Everything needed for a system of logic is there: a general discussion of the nature of signs, a specification of the particular symbols used in logic, a collection of examples of legitimate expressions of the system using the vocabulary, together with their interpretations, laws of the system, and a specification of its logical coefficients.

The heart of the basic logic consists of the three well-known binary 
operators ' $X$ ', ' + ' and '-', and the two constant class symbols 0 and 1 . In modern terms (which are not to be found in Boole's own work), the symbols may be understood as follows: ' $x \times y$ ' stands for the intersection of the classes $x$ and $y$, ' $x+y$ ' for a truncated version of class union in which the expression in question is significant except in those cases in which $x$ and $y$ intersect, and ' $x-y$ ' for what might be called logical exception (i.e., " $x$ 's except for the $\left.y^{\prime} \mathbf{s}^{\prime \prime}\right)$, which is significant only if $y$ is contained in $x$. Of the constants, 0 stands for the null class, and in Laws of Thought, 1 stands for the class containing everything in the universe of discourse.

Equally well-known is the relationship which Boole set up between his basic logic and mathematical algebra. Each of the functions serves as an analogue of its arithmetical counterpart, and the laws of logic correspond in like fashion with expressions in mathematics. Boole circumscribes the extent of the similarity by laying particular stress on the law of idempotence, $x x=x$, which holds universally in the logic, but in standard algebra, only for the values 0 and 1 . This leads Boole to say that "The two systems of elective symbols and of quantity osculate, if I may use the expression, in the points 0 and 1.", That is, "The laws, the axioms, and the processes, of [an algebra in which the symbols $x, y, z$ etc., admit indifferently of the values 0 and 1 and of these values alone] will be identical in their whole extent with the laws, the axioms, and the processes of an algebra of logic. Difference of interpretation alone will divide them.,"12

Toward the end of Chapter II, Boole makes it clear that while each logical operator has an algebraic analogue, the reverse is not the case. There the question of whether division is to be counted as a logical operation arises, and Boole meets it by saying,

... the axiom of algebraists, that both sides of an equation may be divided by the same quantity, has no formal equivalent [in basic logic]. I say no formal equivalent, because, in accordance with the general spirit of these inquiries, it is not even sought to determine whether the mental operation which is represented by removing a logical symbol, $z$, from a combination $z x$, is in itself analogous with the operation of division in arithmetic. That mental operation is indeed identical with what is commonly termed abstraction, and it will hereafter appear that its laws are dependent upon the laws already deduced in this chapter. ${ }^{13}$

In other words, division is not even considered as a possible logical operation since abstraction can be handled with the logical apparatus already at hand, which does not include division. While the question still remains as to whether Boole slips division in later on, his remarks in Chapter II strongly suggest that he was committed to excluding division from consideration as a logical operator.

2 While Chapter II deals with Boole's basic logic, Chapters V ff. introduce a fundamentally different topic. It is within these chapters that virtually all of the expressions with which the critics are concerned appear, and it is here that Boole lays out what he calls a "general method in logic."14 The method differs from the basic logic in the sense that Boole considers the 
earlier logic to be the basis for the generalization which the method will provide:

The previous chapters of this work have been devoted to the investigation of the fundamental laws of the operations of the mind in reasoning; of their development in the laws of the symbols of Logic; and of the principles of expression, by which that species of propositions called primary may be represented in the language of symbols. These inquiries have been in the strictest sense preliminary. They form an indispensable introduction to one of the chief objects of this treatise-the construction of a system or method of logic upon the basis of an exact summary of the fundamental laws of thought. ${ }^{15}$

As the chapter unfolds, the difference between the basic logic and the general method in logic becomes clear. While the basic logic was restricted to dealing with completely interpreted logical operations, the general method involves the application of more generalized formal devices (development being the primary one) in logical contexts. Boole's apparent aim was to be able to use non-logical transformational devices in logical contexts in order to strengthen logical transfor mation.

In describing the general method, Boole begins by asking ". . . whether it is necessary to restrict the application of [symbolical laws of logical processes] by the same conditions of interpretability under which knowledge of them was obtained."16 That is, (using his own example) since the expression ' $x+y$ ' is uninterpretable if $x$ and $y$ do not represent distinct classes, must one only use ' + ' when it is certain that an interpretable expression will result? Boole goes on to say that if such restriction is necessary, no general method is possible. But, "On the other hand, if such restriction is unnecessary, in what light are we to contemplate processes which appear to be uninterpretable in that sphere of thought which they are designed to aid?",17 Not surprisingly, Boole holds such restriction to be unnecessary, and then goes on to answer the question just posed.

He begins by pointing out that in ordinary reasoning, there is always a correspondence between what he refers to as process and interpretation, which means that the various steps in an ordinary reasoning process are all interpretable, i.e., none are merely formal moves. Accordingly, there are those who hold that:

$\ldots$ as the laws or axioms which govern the use of symbols are established
upon an investigation of those cases only in which interpretation is possible,
we have no right to extend their application to other cases in which interpre-
tation is impossible or doubtful, even though (as should be admitted) such
application is employed in the intermediate steps of demonstration only. ${ }^{18}$

Such an objection, says Boole, is fallacious. "It is an unquestionable fact," he says, "that the validity of a conclusion arrived at by any symbolical process of reasoning, does not depend on our ability to interpret the formal results which have presented themselves in the different stages of the investigation."19 This statement forms the foundation of the general method in logic. In concrete terms, Boole reduces the method to the following rule: 
We may in fact lay aside the logical interpretation of the symbols in [a] given equation; convert them into quantitative symbols, susceptible only of the values 0 and 1; perform upon them as such all the requisite processes of solution; and finally restore to them their logical interpretation. ${ }^{20}$

The process Boole is here advocating is of a distinctly different sort from the basic logic of Chapter II. He is suggesting that any logical symbol may be treated as its mathematical counterpart in the manner laid down in Chapter II. Then any available mathematical operation may be used on it, whether that operation is logically interpretable or not. The final (mathematical) expression in the sequence must again be one which corresponds to a logical expression. With the purely mathematical interlude lying between, the sequence may then be treated as the inference of the final (logical) expression from the initial one.

Development plays a central role in this method. Boole considered it to be a process by which one can construct the bridge between logically interpretable expressions in accordance with the above rule. While it performs this logical function, Boole did not consider it to be solely a logical device at all; in fact, he initially defined it as encompassing much more than just logical application:

Definition-Any function $f(x)$ in which $x$ is a logical symbol, or a symbol of quantity susceptible only of the values 0 and 1 , is said to be developed, when it is reduced to the form $a x+b(1-x), a$ and $b$ being so determined as to make the result equivalent to the function from which it was derived.

This definition assumes, that it is possible to represent any function $f(x)$ in the form supposed. ${ }^{21}$

This definition covers any function, logical or not, so long as the variable $x$ may be restricted to the values 1 and 0 . As he puts it later on, the results of development are "true and intelligible for quantitative' symbols (which meet the restrictions mentioned in the definition) always; for logical symbols, always when interpretable.",22 In other words, development need not yield logically significant expressions.

It is at this point that some of the critic's complaints can be dispatched, for some of the allegedly uninterpretable logical symbols to which they point can now be seen to be nothing of the sort. The very first example of development which Boole provides contains several of the expressions in question. In the example, ${ }^{23}$ Boole sets out to develop the function $\frac{1+x}{1+2 x}$, the result being $\frac{1+x}{1+2 x}=\frac{2}{3} x+(1-x)$. But Boole does not claim that this expression is logically interpretable, or is a logical expression in any sense. Rather, he makes it abundantly clear that $x$ is here being treated as a quantitative symbol restricted to the values 0 and 1 , by prefacing the example with the remark that "By the principle which has been asserted in this chapter, it is lawful to treat $x$ as a quantitative symbol, susceptible only of the values 0 and 1."24 The example is properly one of development, but definitely not of development in a logical context.

Excluding consideration of $1 / 0$ and $0 / 0$, to be considered separately 
below, the remaining instances of expressions in LT which the critics claim to be both logical and uninterpretable ${ }^{25}$ fall into one of two classes. First, they may be non-logical expressions which occur in the steps of an inference which intervene between logically interpretable expressions in accordance with the rule of the general method, or second, like the example just considered, they may be constituents of expressions which serve as non-logical examples of processes which also have logical applications. In neither case has Boole done anything obscure, complicated, or unintelligible.

There remains the possibility, however, that Boole illegitimately introduced division as a logical operator. For instance, in providing an example of the development of a function of two variables, Boole uses the function $\frac{1-x}{1-y}$, which, when developed, is $\frac{1-x}{1-y}=\frac{0}{0} x y+0 x(1-y)+$ $\frac{1}{0}(1-x) y+(1-x)(1-y)$. Neither $\frac{0}{0}$ nor $\frac{1}{0}$ have any standing in basic logic, hence we might expect them to be treated as purely mathematical expressions in accordance with the general method as outlined above. Instead, he says that "It will in the next chapter be seen that the forms $0 / 0$ and $1 / 0$, the former of which is known to mathematicians as the symbol of indeterminate quantity, admit, in such expressions as the above, of a very important logical interpretation.,"26

But, in a manner entirely typical of the period, Boole's use of 'logical' is incurably vague and ambiguous. He may have meant that these symbols are, in effect, to be added to basic logic. If this is the proper interpretation, criticisms to the effect that Boole introduced division into logic are justified. But there is an equal likelihood that these expressions were intended to be restricted to the general method, i.e., were meant to apply to, but not be a part of, the expressions of the basic logic. In my view, the latter is the correct interpretation.

The latter interpretation can best be understood by considering another of his examples. Beginning with the statement "Men not mortals do not exist," symbolized $y(1-x)=0$, Boole wishes to provide a "reverse definition" of mortals in terms of men. The initial equation yields, mathematically, $y-y x=0$, or $y x=y$. Of this result he says,

\footnotetext{
Were this an ordinary algebraic equation, we should, in the next place, divide both sides of it by $y$. But it has been remarked in Chapter II that the operation of division cannot be performed with the symbols with which we are now engaged. Our recourse, then is to express the operation, and develop the result by the [general method in logic]. ${ }^{27}$
}

Although this passage is somewhat unclear, owing to the fact that Boole does not say in what the difference between performance and expression consists, presumably he is reaffirming the principle of the general method, whereby division may be used as a step in the transformation process, as long as the process ends with a logically significant expression. Thus "expressing" division yields $x=y / y$ which, again, is not interpretable in basic logic. Boole then develops this equation, the result being

$$
x=y+0 / 0(1-y) \text {. }
$$


This expression, too, seems to be uninterpretable in basic logic, for it contains $0 / 0$, which is not a part of basic logic. But Boole gives $0 / 0$ the following "logical interpretation":

Let [a] coefficient be in the form $0 / 0$. Now, as in arithmetic, the symbol $0 / 0$ represents an indefinite number, except when otherwise determined by some special circumstance, analogy would suggest that in the system of this work the same symbol should represent an indefinite class. $^{28}$

In the particular example under consideration, this interpretation results in the following meaning being assigned to ' $x=y+0 / 0(1-y)$ ': Mortals consist of all men together with an indefinite remainder (some, none, or all) of mortals who are not men.

Before proceeding further, note should be taken of Boole's use of 'some'. Symbolized ' $v$ ', he first introduces its use in Chapter IV. There he refers to it as an elective of a peculiar sort: ". . . it is indefinite in all respects but this, that it contains some individuals of the class to whose expression it is prefixed...", Thus ' $v x$ ' means "some $x$ " or, " $x$ is not empty." While Boole's intent is clear enough, i.e., to provide a device for the expression of existential propositions, the device itself suffers from a fatal flaw, namely, the impossibility of a class whose only defining characteristic is that it have members. The flaw is due largely to the restrictive nature of algebraic logics generally.

With 'some' thus understood, the question now arises, does Boole surreptitiously slip division into his logic via the expression $0 / 0$ ? I am inclined to take his disclaimer concerning division seriously, and suggest that he does not. There are two ways in which the "indefinite remainder" represented by ' $0 / 0$ ' might be understood. ' $0 / 0$ ' might be conceived of as an elective standing for a truly indefinite class, i.e., a class with a literally indefinitely large membership. Were this the intended interpretation, ' $0 / 0$ ' would indeed have to be counted as another (and quite peculiar) variety of elective. Also, on such an interpretation, division would become (implicitly) a part of basic logic.

But it is not at all clear that Boole intended $0 / 0$ to function as a distinct coefficient in basic logic. On the contrary, his use of the symbol in Chapter VI suggests that he thought of $0 / 0$ as being, in effect, a higher level variable ranging over the coefficients already available in basic logic. Specifically, Boole seems to use ' $0 / 0$ ' not to stand directly for an indefinite class, but as a way of indicating the irrelevance of class size, in certain cases, for the validity of inferences in which they occur. For instance, concerning the above example, he asks, ". . . what remainder of not-men is implied by the premise ["men not mortal do not exist"]?" His answer is that ". . . whether those beings which are not men are all, some, or none, of them mortal, the truth of the premise that all men are mortal will be equally unaffected, and therefore the expression here indicates that some, all, or none of the class to whose expression it is affixed must be taken.",30 Although this explanation of the use of ' $0 / 0$ ' is less than totally clear, I take him to be saying, in effect, that the class is indefinite only at a higher 
level. That is, he is saying that the class is composed of some, or none, or all of the mortals, and that there are no other alternatives. Hence at the basic level, the proposition in question is perfectly determinate: it simply falls under one of the three coefficients which the system already provides. The indefiniteness occurs at a higher level, i.e., at the level at which it is determined which of the three possible propositions the class actually contains. In other words, ' $0 / 0$ ' is not simply an addition to ' $v$ ', ' 1 ', and ' 0 ', but is a way of indicating that class size, though definite, need not be specified.

On this interpretation of ' $0 / 0$ ', it need not be considered to be a part of basic logic at all. Rather, it remains a part of the general method only. However, unlike the other constituents of the general method, it is neither simply a purely mathematical part of the method, nor does it have a unique logical counterpart. Instead, it operates at the level of the general method as a variable which ranges over the three logical coefficients.

In summary, in this section I have shown that although there are rough spots in it, and although it defies systematically clear understanding, Boole's logic does not merit much of the criticism aimed it. Charges that Boole includes logically uninterpretable expressions in his logic have been for the most part cleared, in the sense that most of these expressions were understood by Boole to be parts of the general method only, and not logical expressions. While the situation is less clear concerning division, there is at least a way of understanding Boole's use of expressions such as ' $0 / 0$ ' which is consistent with his claim that he does not introduce division as an operation in (basic) logic.

3 Did Boole's successors in algebraic logic really remove the "shortcomings" and "anomalies" from his logic? In the sense that the logics of, e.g., Schröder, Peirce and Whitehead were more fully and systematically developed than Boole's, the answer is "yes." Also, in the later algebraic logics, one finds fewer of the relatively minor technical gaffes of the sort which appear in Boole's work. Thus Peirce and Jevons showed how the restriction which Boole placed on ' + ' could be ignored, and the equivocation on ' $v$ ' which occurs in LT is not found in later algebraic logics.

In a different sense, however, the matter is not as clear. The algebraic logicians in Boole's posterity tended to overlook Boole's device that permits the use of mathematical operations in logical contexts, while avoiding the general conflation of mathematics and logic. Instead, they tend to treat logic and mathematics as being so related that every operation in the one has its unique counterpart in the other, despite the fact that this poses serious problems for logic. They seem to be more interested, that is, in forcing the "fit" between logic and mathematics than in the problems this creates for logic.

Nowhere is this tendency clearer than in the treatment division received at the hands of Boole's logical descendants. While Boole, as indicated earlier, explicitly rejected division as a logical operation in LT, and on the present interpretation avoided even the implicit introduction of the operation into basic logic through such coefficients as $0 / 0$ and $1 / 0$, his 
successors show no reticence at introducing the operation directly into logic. The remainder of this section consists of a few brief examples of how division was handled by some of these successors. In each case, the result of admitting logic as a basic operation is the same, namely, the generation of fundamentally indeterminate classes into (basic) logic.

In a paper entitled "On an Improvement in Boole's Calculus of Logic," C. S. Peirce sets out to ". . . exhibit Boole's system in a modified form." After introducing some minor notational changes, he turns his attention to the topic of logical division:

Let $a ; b$ be read $a$ logically divided by $b$, and be defined by the condition that

$$
\text { If } b, x ; a \quad x ; a ; b
$$

$x$ is not fully determined by this condition. It will vary from $a$ to $a+\bar{b}$ and will be uninterpretable if $a$ is not wholly contained under $b .^{31}$

The inclusion of division in logic has the unfortunate result that if treated as logical abstraction, it yields logically indeterminate classes within what amounts to basic logic itself, which is the result Boole seemingly avoids. That is, $x=a ; b$ is problematic because there is no determination of $b$ with respect to $x$. From the equation, we know that $x$ contains $a$, but how many members of $b$ are members of $x$, if any at all, is left completely undetermined by the operation. Since the analogous indeterminacy in Boole's system was seemingly restricted to a higher level, the same problem did not arise there.

Schröder's Der Operationskreis des Logikkalkuls consists in large part in an attempt to clean up Boole's logic (". . . Nicht deutungsfähigen Symbolen wie 2, $-1,1 / 3,1 / 0$ gerechnet werden muss.",32) In developing his own calculus of logic, he recognizes four basic operations:
Auch in dem kalkul der Logik gibt es, wie in der Arithmetik, 4 Species oder Grundrechnungsarten, welche jedoch, wie sich zeigen wird, endgültig auf $d r e i$ verschiedenartige Elementaroperationen reducirt werden kb̈nnen. Nichts hindert, jene 4 Grundoperationen mit denselben Namen zu benennen und mittelst derselben Rechenzeichen auszudrücken, wie sie in der Arithmetik gebräuchlich sind. ${ }^{33}$

The four operations which Schröder recognizes are (as he sets them out):

\begin{tabular}{llrl}
$\left.1^{\circ}\right) \quad \begin{array}{l}\text { Die Multiplikation, genannt } \\
\text { Determination. }\end{array}$ & $\left.1^{\prime}\right)$ & $\begin{array}{l}\text { Die Addition oder Collektive } \\
\text { Zusammenfassung (Collektion) }\end{array}$ \\
\hline $\left.2^{\circ}\right)$ & Die Division oder Abstraction. & $2^{\prime}$ ) $\begin{array}{l}\text { Die Subtaktion oder Exception } \\
\text { (Ausschliessung). }{ }^{34}\end{array}$
\end{tabular}

Schröder's approach is somewhat different from Peirce's in that he attempts to avoid actively using division in his logic (in [12] he speaks of the "inverse" operations (i.e., division and subtraction) as being primarily of "theoretical interest",35). Nevertheless, he still considers them to be full-fledged operations of the calculus, even if they are "keine unbedingt ausfuhrbaren operationen," due to the fact that unless the usual restrictions are followed, meaningless expressions occur. 
By incorporating division into logic, Schröder meets the same fate as Peirce. Beginning with $c=a \div b$, Schröder determines the value of $c$ in terms of $a$ and $b$ in the following way:

Als Werth von $c$ findet sich nun in der genannten Weise:

$$
\begin{aligned}
a \div b= & a b_{1}+u b \\
& a\left(b_{1}+u\right) \\
& a b_{1}+u a b
\end{aligned}
$$

für ein unbestimmtes $u \ldots .^{36}$

Immediately thereafter, he refers to the class $u$ as "... eine vollig beliebige, arbitrare." Here again, the result of using division as a logical operator is the generation of fundamentally indeterminate classes.

Of Boole's successors, John Venn was particularly familiar with his work. Venn's Symbolic Logic contains perhaps the most complete discussion of Boole's work to be found in the works of any of his successors. Of Venn's work, for instance, Whitehead remarked, "The task of giving thorough consistency to Boole's ideas and notation, with the slightest possible change, was performed by Venn in his 'Symbolic Logic.",37 Early in the book Venn comments that Boole ". . . attempts no explanation whatsoever of his use of the sign of division in logic,",38 and then goes on to ask whether there can be a logical interpretation for the operation which is the inverse of multiplication.

His view, like that of Peirce and Schröder, is that division has a place in logic proper. Venn defines ' $x / y$ ' in terms of what he calls "restriction" in the sense that ' $x / y$ ' is regarded as identifying a class which, when the $y$ restriction is placed on it, yields $x$. The class is hence composed of all $x$, and, of all things which are "... neither $x$ nor $y$, it may take as much or as little as it pleases, that is, a perfectly indefinite proportion." "39 Shortly thereafter he says that it is essential that $x / y$ be regarded as identifying a class, even though it may be ". . . indefinite in regard to its limits . . ., but it is never anything else than a true logical class or conceivably assignable group of individuals." 40

While Boole never gives reasons for his exclusion of division as a logical operation, it is tempting to think that it was occasioned by a desire to avoid such completely indeterminate classes in logic. But regardless of Boole's grounds for rejecting division, suggestions to the effect that Boole's successors did a better job of developing algebraic logic are not entirely convincing. Later logics may have been more tidy, but they lack an important variety of sophistication found in LT. That is, as the preceding discussion indicates, while later logicians were intent on mathematicizing logic, Boole's approach is much more sensitive to the requirements of logic as an independent discipline.

4 There remains the question of Boole's importance within the history of logic. The task of fixing his proper historical place has been largely obscured by critics who measure his achievements, and shortcomings, by contemporary standards. Instances of this sort of criticism can be found among the examples at the beginning of the paper; another is Michael 
Dummett's review of [2]. In his review, he characterizes Boole's achievements by saying,

As had Leibniz two centuries earlier, he devised a general theory of classes under Boolean operations, a theory which of course contained the traditional theory of the syllogism. This move gained its importance for logic rather from the novelty of any extension of logical theory than from the magnitude of the extension itself; and anyone unacquainted with Boole's works will receive an unpleasant surprise when he discovers how ill-constructed the theory actually was and how confused his explanations of it. ${ }^{41}$

Dummett then proceeds to recite the standard criticisms, i.e., those concerning the difficulty of symbolizing statements in algebraic logic, the problems sur rounding ' $v$ ', ' + ', and '-', etc. He also criticizes Boole for not proving that, in effect, the general method is always truth-preserving, and that the process of development really does, as Boole claims it does, follow from Taylor's theorem.

Such criticism is at best uninformative. Boole is simply not a contemporary logician, and measuring him by our current standards serves only to underscore that fact. Since Boole stands on the other side of a major revolution in the science of logic, holding him up to standards used on our side of that revolution is singularly unproductive. That Boole's logic is not as rigorously developed as ours is undeniably true, but also not very significant. Conversely, what is significant are attempts to fix, e.g., the relationship between Boole's work and the logical environment during the period which just preceded the full impact of Frege-Principia logic.

Instead of following the more recent variety of criticism, I have concentrated on a feature of Boole's logic which makes him, I think, an important contributor to the transitional period in logic which occupied much of the nineteenth century. His development of the general method, regardless of its imperfections by our standards, goes well beyond the simple formalization of ordinary language, which had characterized logic from Aristotle's day. In the method, we see one of the first important attempts to bring mathematical methods to bear on logic while retaining the basic independence of logic from mathematics. In this sense, the method constitutes a further step away from the dependence on ordinary language which had characterized earlier logics.

Boole thus stands with a foot in each of two logical epochs; the general method links him with contemporary logic, and the still-present pull of the syllogism with the older epoch. In the transition, moreover, Boole's role is pivotal. After him, algebraic logic, by overly stressing the connection between logic and mathematics, headed toward extinction by losing sight of logic as an independent discipline altogether.

\section{NOTES}

1. By R. Feys in "Boole as a logician," Proceedings of the Royal Irish Academy, vol. 57, Sec. A, No. 6, pp. 97-106. 
2. E.g., "Boole cannot correctly be called the "father of modern logic'." Michael Dummett in [11], p. 205.

3. A particularly engaging assessment of Boole's general contribution to logic was given by Hugh MacColl:

[The Mathematical Analysis of Logic and Laws of Thought] excited much admiration in the mathematical world, and, it may be added, caused no small trepidation among logicians, who saw their hitherto inviolate territory now for the first time invated by a foreign power, and with weapons which they had too much reason to dread. ("Symbolical reasoning," Mind, vol. V (1880), p. 46.)

4. [5], p. 113. As early as 1864, Jevons displayed a clear understanding of Boole's general method in logic, explained below. Thus in [6], (\$176, pp. 67-68), he accurately describes Boole's process, but rejects it as not corresponding "to the laws and conditions of thought in reality."

5. [7], p. 115.

6. [3], p. 298 .

7. [8], p. 422 .

8. [9], p. 171.

9. [4], p. 543.

10. Boole never published the essay in question and Rhees ([2], p. 12) quotes De Morgan as saying that Boole would not have wished such an essay published posthumously, since it might give the impression that LT had been rejected. Also, in the essay, Boole seems to accept division as a logical operation; however, in a later published work (Differential Equations, Supplementary volume, MacMillan \& Co., Cambridge (1865)) he again denies (p. 377) that inverse symbols stand for operations. In addition, Venn was of the opinion that Boole never included inverse operations in his logic. (Cf. [15], p. 77n.)

11. [2], p. 119.

12. [1], pp. 37-38.

13. [1], pp. 36-37.

14. Boole refers to the process in a number of ways, and never gives it a definite name. I have chosen "general method in logic" as a matter of convenience. Boole uses the term in [1], p. 70, when he says that if logical symbols were restricted to logically interpretable contexts only, "... the inquiry after a general method in Logic would be a hopeless quest."

15. [1], p. 66 .

16. [1], p. 67.

17. Ibid. Jevons held such a view, though it is unlikely that Boole was referring to him.

18. [1], p. 67 .

19. [1], pp. 67-68.

20. [1], p. 70. Boole italicized the entire passage. 
21. [1], p. 72 .

22. [1], p. 77 .

23. [1], p. 73 .

24. [1], p. 72 .

25. A fairly complete list of these as they occur in Chs. V-XV of Laws of Thought is as follows: (in each pair the first number is the page, and the expression following is the supposedly uninterpretable symbol occurring there) $94,-1 ; 95, \frac{1}{2}$; $97, \frac{1}{2} ; 115,2 ; 123,2 ; 125,2 ; 131,2$.

26. [1], p. 74 .

27. [1], p. 89 .

28. Ibid.

29. [1], p. 63 .

30. [1], pp. 89-90.

31. [12], \$3.6 (p. 6). Here ' $a, b$ ' stands for the logical multiplication of $a$ and $b$. ' $a ; b$ ' stands for the "numerical identity" of the classes $a$ and $b$.

32. [13], p. iii.

33. [13], p. 2.

34. Ibid.

35. $C f$. [14], Vol. I, pp. $478 \mathrm{ff}$.

36. [13], p. 30 .

37. [16], p. 115.

38. [15], p. viii.

39. [15], pp. 77, 78 .

40. [15], p. 79 .

41. [11], p. 205.

\section{REFERENCES}

[1] Boole, George, An Investigation of the Laws of Thought, Dover Publications, Inc., New York (1951). A reprint of the London edition of 1854.

[2] Boole, George, Studies in Logic and Probability, edited by R. Rhees, Watts \& Co., London (1952).

[3] Bocheński, I. M., A History of Formal Logic, University of Notre Dame Press, Notre Dame, Indiana (1961). A revised translation (by I. Thomas) of Formale Logik, Verlag Karl Alber, Frieburg, Munich (1956).

[4] Heath, P. L., "Boole," in The Encyclopedia of Philosophy, The MacMillan Co. and The Free Press, New York; Collier-MacMillan, London (1967). 
[5] Jevons, W. Stanley, The Principles of Science, a Treatise on Logic and Scientific Method, Dover Publications, Inc., New York (1958). A reprint of the London edition of 1874 .

[6] Jevons, W. Stanley, "Pure Logic" (1864) in Pure Logic and Other Minor Works, Burt Franklin, New York (1971). A reprint of the London edition of 1890.

[7] Jorgensen, Jorgen, A Treatise of Formal Logic, Russell and Russell, New York (1962). A reprint of the Copenhagen edition of 1931.

[8] Kneale, William, and Martha Kneale, The Development of Logic, Oxford University Press, Oxford, England (1962).

[9] Kotarbiński, Tadeusz, Leçons sur l'histoire de la logique, Presses universitaires de France (1964). A translation of Wykłady $z$ dziejów logiki, PWN, Warsaw (1964).

[10] Lewis, C. I., A Survey of Symbolic Logic, Dover Publications, Inc., New York (1960). A revision of the University of California Press edition of 1918.

[11] Dummett, Michael, Review of [2], The Journal of Symbolic Logic, vol. 24 (1959), pp. 203-209.

[12] Peirce, Charles S., Collected Papers of Charles Sanders Peirce, edited by Charles Hartshorne and Paul Weiss, Harvard University Press, Cambridge (1965).

[13] Schröder, E., Der operationskreis des logikkalkuls, Teubner, Leipzig (1877).

[14] Schröder, E., Vorlesungen uber die algebra der logik, 3 vols., Chelsea Publishing Co., Bronx, New York. A reprint of the Leipzig edition of 1890-1905.

[15] Venn, John, Symbolic Logic, Chelsea Publishing Co., Bronx, New York (1971). A reprint of the London edition (2nd) of 1894.

[16] Whitehead, A. N., A Treatise on Universal Algebra, Hafner Publishing Co., New York (1960). A reprint of the Cambridge edition of 1897.

University of Waterloo

Waterloo, Ontario, Canada 